\title{
A influência das percepções de benefícios, resultados e dificuldades dos grupos de pesquisa sobre as interações com empresas*
}

Veneziano de Castro Araujo **

Suelene Mascarini***

Emerson Gomes dos Santos****

Ariana Ribeiro Costa ${ }^{* * * * *}$

Resumo

O presente trabalho tem por objetivo investigar como as percepções dos grupos de pesquisa acadêmica sobre resultados, benefícios e dificuldades esperados das interaçóes influenciam o número de interaçôes que eles realizam com empresas, com base em um survey sobre as relações universidade-empresa no Brasil. Para tanto, por meio da Teoria de Resposta ao Item não Paramétrico (TRIN), foram criados agrupamentos não ad hoc a partir de padrões de resposta de pesquisadores para as percepções analisadas. A partir dos agrupamentos, foi estimado um modelo para identificar como essas percepçóes influenciam o número de interaçôes dos grupos. Os resultados apontam que os grupos de pesquisa que percebem benefícios intangíveis e

* $\quad$ Os autores agradecem o apoio financeiro recebido pela Fapesp através do projeto "Geografia e Inovação: uma análise dos efeitos da aglomeração industrial e suas implicações de políticas" e do Cnpq através do projeto "Interação Universidade-Empresa

** Universidade de São Paulo (USP). Atualmente professor da Universidade Federal de São Paulo (Unifesp), Osasco (SP), Brasil. E-mail: venezianoaraujo@unifesp.br

*** Universidade de São Paulo (USP), São Paulo (SP), Brasil. E-mail: smascarini@gmail.com

**** Universidade de São Paulo (USP). Atualmente professor da Universidade Federal de São Paulo (Unifesp), Osasco (SP), Brasil. E-mail: emerson.gomes@unifesp.br

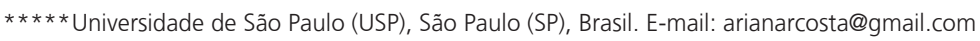


resultados de conhecimentos como mais importantes tendem a apresentar maior número de interações com empresas. Além disso, as dificuldades transacionais implicam menos interações com empresas. Por fim, são tecidos alguns desdobramentos para políticas públicas.

PALAVRAS-CHAVE | Interação Universidade-Empresa; Conhecimento e Inovação; Economia da Tecnologia.

Códigos JEL: O31; O32; O33

\title{
Influence of benefits, results and obstacles' perceptions by research groups on interactions with companies
}

\begin{abstract}
The aim of this paper is to investigate how expected perceptions of academic research groups about results, benefits and obstacles influence the number of interactions with firms, based on a survey of university-industry interactions in Brazil. For this purpose, by means of a nonparametric Item Response Theory (NIRT), non ad hoc clusters were created from patterns of survey answers related with the analyzed perceptions. Using these clusters, a model was estimated to identify how perceptions influence the number of interactions of research groups. The results indicate that research groups that perceive intangible benefits and knowledge results as more important tend to have more interactions with firms. In addition, transactional obstacles imply in less interactions with firms. Finally, some implications on public policies are presented.
\end{abstract}

KEYWORDS | University-Industry Linkages; Knowledge and Innovation; Economics of Technology.

JEL-CODES | O31; O32; O33 


\section{Introdução}

O papel da universidade está além da formação de pessoal. A universidade tem sido apontada como um importante agente no processo inovativo, por meio da transferência de propriedade intelectual, do empreendedorismo acadêmico, do desenvolvimento de bases científicas de vanguarda, entre outras formas de relacionamento.

Assim, mesmo com uma dinâmica distinta das empresas, as universidades são importantes agentes inovativos e também podem ser beneficiadas com as interaçōes entre universidades e empresa (U-E). Para ambas, a interação tende a facilitar o fluxo de conhecimento, gerar novas possibilidades de projetos e intercâmbios e permitir novas agendas de pesquisa.

As interações U-E já foram avaliadas em diversos estudos acadêmicos. Alguns focaram o impacto das interaçôes nos processos inovativos ou o seu papel para a pesquisa universitária (KLEVORICK et al., 1995; NELSON, 1996; COHEN et al., 2002). Outros trabalhos analisaram os fatores que afetam a interação e o modo como os agentes envolvidos avaliam tais relações e seus possíveis benefícios, resultados e dificuldades (ARZA, 2010; ARZA; VASQUEZ, 2010; TARTARI;BRESCHI, 2012).

Nesse contexto, o presente artigo tem o objetivo de investigar como as percepções dos grupos de pesquisa acadêmica sobre resultados, benefícios e dificuldades esperados das interações influenciam o número de interações que eles realizam com empresas. Esse tema foi abordado em trabalhos como Tartari e Breschi (2012), Tartari et al. (2012), D'Este e Perkmann (2011), Arza e Vazquez (2010) e Fernandes et al. (2010).

Para tanto, são utilizados dados de um survey realizado no Brasil, em 2008, sobre as interações entre universidades e empresas. Além dos aspectos relacionados às percepções dos grupos de pesquisa acadêmicos, são considerados também fatores ligados às características dos grupos, como a qualidade da pesquisa acadêmica e o tamanho do grupo de pesquisa e do departamento ao qual o grupo pertence.

Duas contribuições desse estudo podem ser apontadas para a literatura. A primeira é a identificação de padrões de percepções dos grupos de pesquisa relacionados a resultados, benefícios e dificuldades esperados ao interagir. Este resultado é derivado da aplicação de uma metodologia denominada Teoria de Resposta ao Item não paramétrica (TRIN), frequentemente utilizada para tratamento de dados declarados em questionário, pois possibilita a identificação dos padrōes por meio da geração de escalas (agrupamentos dos itens do questionário) não subjetivas, derivadas das respostas obtidas. Ou seja, é possível criar agrupamentos validados empirica- 
mente que permitem uma análise mais segura das relações entre os diferentes tipos de resultados, benefícios e dificuldades das interações.

A segunda contribuição é subsidiar o debate sobre como as percepções influenciam as interaçôes dos grupos de pesquisa com dados brasileiros. A partir das escalas geradas pela TRIN é realizada uma análise inferencial por meio de um modelo econométrico. Esse passo se mostra importante para a literatura, pois, de acordo com Tartari e Breschi (2012), os estudos sobre as interaçôes U-E dessa natureza ainda são incipientes para países em desenvolvimento. Além disso, Tartari et al. (2012) sugeriram que a análise da decisão de interagir deve levar em conta que a interação é complexa e multifacetada, o que exige julgamento conjunto dos fatores que a influenciam, como custos e benefícios.

$\mathrm{Na}$ seção a seguir, são apresentadas as principais bases conceituais sobre a importância das universidades nas interações U-E e as percepções sobre os resultados, benefícios e dificuldades esperados para sua realização, além de um breve levantamento das especificidades das interaçōes no Brasil. Em seguida, expõem-se os dados e as informações que permitiram identificar padrôes de respostas nos itens dos questionários e, assim, classificá-los em diferentes agrupamentos para as dimensões analisadas - resultados, benefícios e dificuldades. Posteriormente, apresentam-se os agrupamentos das percepçóes analisadas e detalham-se o modelo e seus resultados. Por fim, são ponderadas e discutidas as conclusōes e algumas implicações de políticas.

\section{Revisão da literatura}

A inovação está longe de ser um processo isolado dentro das empresas. Diversos estudos apontaram a relevância de agentes externos que reforçam as estruturas internas de $\mathrm{P} \& \mathrm{D}$ das firmas, como os fornecedores, os usuários e a universidade (NELSON, 1996; MOWERY et al., 2004). A aproximação dessas fontes foi, em grande parte, derivada do aumento da complexidade dos conhecimentos requeridos para a inovação nas empresas. Dessa forma, a pesquisa acadêmica - e assim a universidade - configurou-se como uma importante fonte de novos conhecimentos tanto para as empresas como para a sociedade em geral (KLEVORICK et al., 1995; ROSENBERG; NELSON, 1994).

Em seu cerne, a universidade possui duas finalidades primordiais no apoio à inovação: a geração de novos conhecimentos, por meio da pesquisa científica; e a formação de trabalhadores qualificados. A pesquisa científica e as atividades de de- 
senvolvimento realizadas diretamente pela universidade contribuem para a geração de novos conhecimentos e, dessa forma, para o progresso científico e tecnológico da sociedade. Já os cientistas e engenheiros oriundos de suas cadeiras colaboram diretamente para os processos inovativos das empresas, por meio da aplicação do conhecimento e de habilidades adquiridas nessas instituições.

Desse modo, trabalhos como os de Klevorick et al. (1995) e Cohen et al. (2002) mostram que a universidade tem assumido papel crescente no fomento às atividades inovativas das empresas. Klevorick et al. (1995) apontam que os novos conhecimentos gerados na universidade são necessários para o desenvolvimento industrial e especialmente importantes em setores considerados de alta tecnologia, devido à maior proximidade à base de conhecimentos científicos. Além disso, segundo Cohen et al. (2002), a universidade afeta de modo importante a Pesquisa e Desenvolvimento (P\&D) em diversos setores e tem desempenho decisivo no fomento da inovação em algumas indústrias. Ainda de acordo com esses autores, as interaçôes entre universidade e as áreas de P\&D das empresas são capazes de sugerir novos projetos de pesquisa industrial, como também colaborar com a conclusão de projetos em andamento.

Ainda que se compreenda a importância das interaçōes tanto para as universidades como para as empresas, em função, principalmente, da natureza de cada um desses agentes, a perspectiva da interação pode ser bastante distinta entre eles.

Em geral, mesmo com o desenvolvimento de pesquisas mais profundas e de longo prazo (ROSENBERG, 1990), as atividades das empresas estão ligadas à busca de resultados de curto prazo, baseados nas necessidades de produção e de comercialização de seus produtos. Já a universidade desenvolve conhecimento com um caráter público, aberto à ampla divulgação e discussão, principalmente via publicaçōes acadêmicas e apresentaçōes em congressos e seminários (MERTON, 1973; DASGUPTA; DAVID, 1994; MEYER-KRAHMER; SCHMOCH, 1998). De tal forma, existem diferentes orientações que conduzem o trabalho dentro das universidades e das empresas que fazem com que as percepções e as formas de se realizarem as interações sejam distintas (BEKKERS; FREITAS, 2008).

Especificamente, autores como Dasgupta e David (1994) apontam que as empresas e a academia possuem estruturas institucionais de incentivos distintas, que levam a padrōes de resultados diferentes, rotulados pelos autores como "Tecnologia Proprietária" e "Ciência Aberta". A primeira está vinculada às pesquisas industriais e envolve intercâmbio limitado de conhecimento, podendo acarretar adiamento de publicaçôes dos resultados científicos. Já a "Ciência Aberta", associada à visão de 
Merton (1973), trabalha com princípios de divulgação e publicação do conhecimento. Segundo os autores, o intercâmbio entre a "Ciência Aberta" e a "Tecnologia Proprietária” exige reconciliar, de alguma maneira, normas e mitigar suas diferenças. Alguns mecanismos mais frequentes de intercâmbio entre universidade e empresa, citados pelos autores, são a contratação pelas empresas de pesquisadores acadêmicos, a mobilidade de pessoal entre esses dois ambientes e as relações diretas entre universidade e empresas. Essas últimas implicam que ambas as partes precisam concordar em objetivos da pesquisa e regras de publicação e podem se configurar de diferentes maneiras.

Nesse sentido, a avaliação das interações pelos grupos de pesquisa e pelas empresas é distinta e as percepções a cerca dos benefícios, resultados e dificuldades da interação se tornam importantes elementos para a consolidação das interações. Esse tópico foi explorado por Arza (2010), Bishop et al. (2011), Tartari et al. (2012), Tartari e Breschi (2012), D'Este e Perkmann (2011), entre outros. Por exemplo, Tartari e Breschi (2012) investigaram como as percepções dos pesquisadores sobre benefícios e dificuldades esperados das interações impactam sua decisão de interagir com empresas.

Em relação aos benefícios, Arza (2010) argumentou que, do ponto de vista das universidades, existem dois tipos distintos de benefícios: os intelectuais, que são aqueles relacionados aos processos de compartilhamento de informações e conhecimentos, reputação e ideias para novas pesquisas e projetos; e os econômicos, que compreendem o compartilhamento de instrumentos e pessoal, os ganhos de recursos financeiros e o fornecimento de insumos para pesquisa. ${ }^{1}$

Arza e Vasquez (2010), avaliando os mesmos benefícios, apontaram que ambos são vistos como importantes pelos pesquisadores, sendo que os benefícios intelectuais são mais bem avaliados em termos de relevância para a interação. Já Tartari e Breschi (2012) encontraram evidências de que o acesso a recursos para a pesquisa (não apenas fundos, mas também equipamentos, aumento da visibilidade acadêmica e a possibilidade de novas ideias de pesquisa) influi positivamente na ocorrência da interação. Em consonância, Perkmann e Wash (2009) argumentam que, para as universidades, os projetos de pesquisa conjunta são importantes meios de trocas de conhecimentos e frequentemente resultam em publicações acadêmicas. A pesquisa

1 Esse trabalho tem foco na ótica das universidades. Do ponto de vista das empresas, os benefícios ocorrem por mecanismos distintos relacionados à estratégia de inovação e à solução de problemas produtivos. Conforme Bishop et al. (2011), as interaçōes U-E permitem às empresas explorar o estoque disponível de conhecimento na universidade. Nas atividades de solução de problemas de curto prazo, a interação amplia a capacidade das empresas de resolver problemas reais, por meio do fornecimento de treinamento para graduados e do desenvolvimento de novos instrumentos e métodos. 
contratada e a consultoria, ainda que possuam relações com objetivos mais aplicados (gerando assim menos publicaçóes), podem propiciar benefícios indiretos para a universidade, como a criação de novas ideias e agendas de pesquisa.

Para Garret-Jones et al. (2010), os pesquisadores, ao se engajarem em colaborações com as empresas, levam em conta suas motivações intangíveis, como melhor acesso a parcerias industriais e a possibilidade de trabalhar com outros pesquisadores. Isto é, para os pesquisadores, muitas vezes, a interação constitui importante fator para suas carreiras na universidade e para o seu desenvolvimento acadêmico. Segundo D’Este e Perkmann (2011), alguns benefícios da interação são de difícil mensuração direta, uma vez que nem sempre levam a resultados científicos concretos. Alguns benefícios, também considerados intangíveis para esses autores, podem levar a novos tópicos de pesquisa e ao aprendizado sobre aplicações industriais, o que é de grande relevância para algumas áreas de conhecimento, como a Física e as Engenharias.

No entanto, não são somente as perspectivas positivas que afetam as interações U-E. As percepções atreladas às suas dificuldades também se configuram como importante fator para a consolidação dessas parcerias. Para Tartari e Breschi (2012), a percepção de perda de liberdade acadêmica e a preocupação com relação ao sigilo da pesquisa são alguns fatores que reduzem a propensão dos pesquisadores a interagir.

Nessa linha, Tartari et al. (2012) afirmam que os pesquisadores, antes de realizarem uma interação, medem também os possíveis custos dessa atividade, divididos pelos autores em dois tipos de obstáculos: os mertonianos, em menção ao trabalho de Merton (1973), que são aqueles associados às diferenças de orientação da pesquisa na academia e nas empresas; e os obstáculos de Williamson, alusão ao trabalho de Williamson (1987), que se referem aos custos de transação da interação, provenientes de entraves em processos burocráticos e institucionais, de políticas de transferência tecnológica, de conflitos de propriedade intelectual, entre outros.

Tartari et al. (2012) encontraram evidências de que os pesquisadores percebem como mais expressivas as barreiras associadas às diferenças de orientação da academia e das empresas, ou seja, os obstáculos mertonianos. Para as barreiras transacionais, os autores concluíram que não existe uma relação tão evidente. Pode-se constatar que a percepção do pesquisador sobre as barreiras à interação é dependente da experiência acadêmica em interagir com a indústria. Isto é, o pesquisador tende a perceber maiores barreiras quando não interage com frequência, o que aumenta a dificuldade de diálogo com as diferentes divisões administrativas da universidade. 
A falta de flexibilidade e a pequena atuação dos escritórios de transferência de tecnologia da universidade são indicativas desse tipo de dificuldade.

Ainda segundo esses autores, a atuação de um grupo de pesquisa em diferentes canais de interação (como consultorias, pesquisa conjunta, treinamento, entre outros) e com distintas aplicações industriais tende a elevar as barreiras transacionais, uma vez que cada tipo de interação exige conhecimento específico sobre os trâmites dos processos dentro da universidade, o que acaba aumentando a percepção sobre essas barreiras. Além disso, a confiança entre os agentes envolvidos na interação é um fator de extrema importância, capaz de reduzir a percepção de ambas as barreiras.

Como apontam Audrestch et al. (2002), problemas burocráticos também podem inviabilizar os processos de interação. Isto é, ainda que a alta administração universitária esteja comprometida com parcerias com o setor industrial, a burocracia nos níveis operacionais pode dificultar a realização das interaçôes.

Além das motivações e percepções dos grupos de pesquisa, suas próprias características podem configurar-se como fatores que influenciam a quantidade de interaçôes realizadas com empresas, como a qualidade da pesquisa acadêmica, os recursos disponíveis e as diferenças relacionadas às áreas de conhecimento.

De acordo com Mansfield e Lee (1996), a excelência acadêmica é um fator de estímulo à cooperação com as empresas, principalmente quando os problemas tecnológicos da firma estão mais próximos da fronteira do conhecimento. Para Tornquist e Kallsen (1994), as instituições de pesquisa mais qualificadas tendem a produzir mais facilmente conhecimento com aplicação industrial. Outros trabalhos, como Bishop et al. (2011), D’Este e Iammarino (2010) e Laursen et al. (2011), também abordaram como a qualidade da pesquisa acadêmica influencia as interações.

Autores como Mansfield e Lee (1996), Schartinger et al. (2001), Perkmann et al. (2011) e De Fuentes e Dutrénit (2012) apontam também que o tamanho do departamento universitário ou da equipe de pesquisa se apresenta como outro fator importante para a análise dessas interaçôes. De acordo com Schartinger et al. (2001), departamentos maiores tendem a possuir mais recursos humanos, físicos e financeiros disponíveis para a pesquisa, além de um maior estoque de conhecimento e de competências para a interação. Desse modo, grupos situados nesses departamentos são mais propensos a interagir, ou seja, devem possuir maior número de interações do que grupos pertencentes a departamentos menores, com menor estrutura de apoio às interaçôes. Além disso, segundo De Fuentes e Dutrénit (2012), grupos de 
pesquisa que possuem maiores estruturas de pessoal são capazes de deter capacitações acadêmicas mais amplas e, em diversos casos, mais diversificadas, o que também influencia sua capacidade de interação.

Para Metcalfe (2003), a natureza das áreas de conhecimento e suas formas específicas de acumulação de conhecimentos variam de acordo com as distintas disciplinas. Áreas de Engenharia e de tecnologias aplicadas estão mais associadas à acumulação de conhecimento do tipo "tentativa e erro" e voltado para a solução de problemas. Por isso, os grupos de pesquisa dessas áreas tendem a interagir mais com as empresas em comparação com os grupos das demais ciências básicas. Assim, de acordo com Schartinger et al. (2001), grupos de pesquisa de campos tecnológicos mais aplicados interagem, em média, mais do que os de outras áreas.

Além das características dos grupos, outros fatores externos, como o nível de urbanização, a estrutura industrial e a qualificação da mão de obra da região em que os grupos se localizam, podem moldar suas interações. Grupos universitários situados em regiões urbanas mais adensadas podem ser beneficiados pelas maiores possibilidades de interagir, relacionadas aos maiores fluxos locais de conhecimento e às possibilidades de inovação, como apontam os trabalhos de Storper e Venables (2004) e Duranton e Puga (2001). Dessa forma, a análise dos fatores que afetam as interações entre universidades e empresas deve considerar fatores internos e externos aos grupos de pesquisa.

\subsection{Interações U-E no Brasil}

Diversos trabalhos foram desenvolvidos na tentativa de compreender os padrões de interação entre universidade e empresas e suas especificidades para o caso brasileiro (SUZIGAN et al., 2009; RAPINI et al., 2009; FERNANDES et al., 2010; SUZIGAN; ALBUQUERQUE, 2011; CHAVES et al., 2012).

De acordo com Suzigan et al. (2009), as universidades brasileiras têm importante papel na criação e difusão de conhecimento entre empresas. As interações U-E realizadas beneficiam ambas as partes, sendo uma importante fonte de processos inovativos para as empresas e de criação de novos projetos de pesquisa e novas questôes de investigação para a universidade.

As interações caracterizam-se por dois importantes mecanismos de transferência de conhecimento: a transmissão de conhecimento codificado por meio de serviços e treinamento; e a criação de fluxos bidirecionais de informação e conhecimento 
por meio da colaboração em projetos de $\mathrm{P} \& \mathrm{D}$ que envolvam pesquisadores das empresas e da universidade. Os autores também apontam para a baixa participação dos escritórios nas interações.

Para a análise dos benefícios das interações U-E brasileiras, aos moldes de Arza (2010), Fernandes et al. (2010) avaliaram os canais de interação que levam a diferentes percepções de benefícios para as empresas, universidade e institutos de pesquisa do país. Os autores apontaram que distintos canais de interação (canal tradicional, de serviços, comercial e bidirecional ${ }^{2}$ ) fornecem diferentes tipos de benefícios para os pesquisadores, o que influencia na decisão de interagir.

Os resultados dos autores indicam que, na percepção da universidade, os canais bidirecionais são mais importantes para a interação, uma vez que induzem a benefícios econômicos e intelectuais. Segundo os autores, os resultados encontrados são condizentes com a configuração de um Sistema Nacional de Inovação ainda imaturo, mas que já possui trocas de conhecimento entre partes distintas e produção de benefícios inovativos e intelectuais.

Além disso, é importante ter em conta as especificidades nacionais nos estudos de interaçōes U-E. Como mostram Nelson (1996) e Freeman (1995), a existência de importantes especificidades nacionais indica que os objetivos dos agentes envolvidos no processo de inovação, e assim da interação entre empresas e universidade, são específicos de cada país e estão fortemente relacionados às questôes históricas e ao estágio de desenvolvimento nacional.

Contudo, a despeito dos estudos sobre as interações U-E no Brasil, análises sobre como as percepçóes dos benefícios, dificuldades e resultados afetam as interação ainda são escassas. ${ }^{3}$ Novos trabalhos podem subsidiar uma análise mais aprofundada sobre as interaçóes U-E no Brasil e ajudar a tecer melhor as políticas públicas sobre o tema.

\section{Dados utilizados}

Para avaliar o objetivo proposto, ou seja, investigar como as percepçóes dos grupos de pesquisa acadêmica sobre resultados, benefícios e dificuldades esperados das interaçôes influenciam o número de interações que eles realizam com empresas,

2 Canal tradicional refere-se às formas tradicionais de interação, como publicaçôes e conferências; o canal de serviços está ligado à troca financeira de serviços técnicos e científicos; o comercial relaciona-se com a comercialização de tecnologia da academia, advinda de seus esforços de pesquisa próprios, como patentes, start-ups e incubadoras; e o canal bidirecional inclui projetos de P\&D contratados e conjuntos, com interações de longo prazo orientadas para a criação de conhecimento e inovação.

3 Um trabalho que analisa as percepções das interaçôes U-E para o caso brasileiro é o de Fernandes et al. (2010). 
foram utilizadas informações de um survey realizado junto aos grupos de pesquisa brasileiros que declararam ter interaçôes com empresas. As questôes do survey continham, além da percepção dos resultados, benefícios e dificuldades das interações, as principais características dos grupos de pesquisa, como a qualificação e o número dos integrantes dos grupos, a área do conhecimento de atuação e a instituição à qual o grupo está filiado.

A aplicação do questionário foi realizada em 2008 e envolveu grupos de pesquisa de todo o país que declararam possuir interações com empresas no Censo de 2004 do Diretório dos Grupos de Pesquisa do CNPq. Os questionários foram respondidos, por meio de um formulário eletrônico, pelos líderes dos grupos de pesquisa selecionados. ${ }^{4}$

Foram enviados 2.151 questionários e, desse total, 1.005 respostas válidas foram recebidas. Aos dados do survey (1.005 respostas), a partir da instituição na qual o grupo estava filiado, foram adicionadas informaçôes sobre a qualidade da pesquisa acadêmica e o tamanho do departamento dos grupos.

Como medida da qualidade da pesquisa acadêmica, utilizou-se a nota do programa de pós-graduação ao qual o grupo de pesquisa está associado. ${ }^{5}$ Ainda que não compreenda todos os tipos de instituição e não capte todas as nuances da qualidade acadêmica, este indicador é comparável e semelhante aos utilizados em outros trabalhos (LAURSEN et al., 2011; D'ESTE; IAMMARINO, 2010; ABRAMOVSKY et al., 2007). Para o tamanho do departamento do grupo, empregou-se como medida o total do número de docentes que estão associados a algum programa de pós-graduação na unidade da instituição que o grupo pertence. Esses dados foram obtidos pela base GeoCapes do ano de 2007. ${ }^{6}$

Além disso, foram acrescentadas informações sobre a qualificação da mão de obra e a densidade urbana da microrregião onde os grupos estão localizados, uma vez que esses fatores exógenos podem influenciar o número de interações. O principal objetivo da inclusão dessa informação é controlar eventuais diferenças da qualificação da mão de obra da região do grupo de pesquisa, uma vez que os grupos

4 Esse survey foi realizado no âmbito da pesquisa "Interaçōes de Universidades e Institutos de Pesquisa com Empresas no Brasil", que teve apoio financeiro da Fapesp, CNPq e do International Development Research Center do Canadá.

5 As notas são disponibilizadas pela Capes para cada área de conhecimento e seguem uma escala de 1 a 7 . Para este trabalho, foram utilizadas as notas do triênio 2004-2006.

6 Para isso, utilizou-se a Instituição de Ensino Superior (IES). A título de exemplo, para a Capes, no triênio analisado, a Unicamp seria dividida em duas unidades de pós-graduação: Unicamp e Unicamp/PIR. GeoCapes é o portal de dados estatísticos georreferenciados disponíveis da Capes. 
acadêmicos situados em regiōes urbanas mais adensadas podem ser beneficiados por maiores possibilidades de interação.

Após o acréscimo dessas informações, optou-se por analisar os dados completos que correspondem a 612 grupos de pesquisa de todo Brasil. Esses grupos pertencem a 79 universidades de todas as regióes brasileiras e de todas as áreas de conhecimento. Em conjunto, os grupos analisados realizaram 2.771 interações com empresas de diversos setores econômicos.

As três dimensões das percepções sobre as interações U-E (resultados, dificuldades e benefícios) utilizadas neste trabalho foram coletadas a partir de uma lista de itens predefinidos no survey. Cada um desses itens deveria ser avaliado pelo respondente de acordo com sua percepção do grau de importância em cada dimensão (sem importância, pouco importante, moderadamente importante ou muito importante).

Ainda, dois pontos importantes devem ser ressaltados. As percepções de benefícios e resultados da interação possuem elementos semelhantes, o que leva ao uso dessas dimensões de forma indistinta em trabalhos sobre o tema. Assim, há uma limitada capacidade de comparação e, até mesmo, de interpretação das evidências entre diferentes análises sobre o tema. Neste trabalho, há uma linha de separação entre as dimensōes benefícios e resultados.

Um elemento-chave para entender essa distinção está relacionado ao alcance dessas percepçóes. Os benefícios da interação podem ser alcançados pelos pesquisadores de modo independe do resultado da interação, já que uma interação com resultado final falho (que não gerou uma tese, uma publicação ou uma nova descoberta científica, por exemplo) pode gerar benefícios para um grupo, como a criação de redes de relacionamento e novas ideias de projetos e pesquisa. Ou seja, em linha com a formulação do questionário utilizado no survey, entende-se que os resultados da interação estão mais relacionados aos objetivos finais esperados da interação, tais como publicaçôes, novos projetos e descobertas científicas, teses, entre outros. Já os benefícios estão associados ao processo de interação e ganhos oriundos dessas atividades, como fundos adicionais para pesquisa, acesso a equipamentos e ideias para pesquisa ou projetos.

Em segundo lugar, é importante também ressaltar que o questionário foi elaborado para captar a percepção geral sobre o relacionamento do grupo com empresas. Nesse sentido, as perguntas não vinculam as respostas a uma interação específica, mas sim ao que o pesquisador espera de suas interaçôes como um todo. 


\section{Metodologia utilizada para agrupamentos dos itens do questionário}

Com a finalidade de avaliar como as percepções dos grupos de pesquisa acadêmica sobre resultados, benefícios e dificuldades esperados das interaçôes influenciam o número de interaçōes que eles realizam com empresas, foram identificadas escalas (ou agrupamentos dos itens) que definem cada uma das dimensões estudadas, a partir dos padrões de respostas.

Tal procedimento foi realizado com o intuito de reduzir a quantidade de itens para cada dimensão, além de evitar problemas de multicolinearidade no modelo estimado, algo que poderia ocorrer ao se escolherem de forma ad hoc os itens para compor cada dimensão. Assim, o modelo estimado reflete os parâmetros de forma mais adequada e fornece estimativas apropriadas para as interpretações dos coeficientes do modelo.

Dessa forma, foi utilizada uma técnica denominada Teoria de Resposta ao Item Não-Paramétrica (TRIN), que se apresenta como um instrumento apropriado para essa análise, pois permite identificar escalas (agrupamentos) a partir do padrão de respostas que um indivíduo produz por meio de questionários. ${ }^{7}$ Essa técnica gera agrupamentos ex post a partir desses padrôes, o que evita incluir arbitrariedades na seleção de grupos de variáveis, necessidade apontada por Fernandes et al. (2010).

Assim, o objetivo da TRIN é selecionar grupos de itens que formem uma escala coerente, ou seja, que os itens agrupados apresentem adequado índice de escalonabilidade (Índice H), com nível de significância de 5\%. ${ }^{8}$ Os resultados da aplicação da TRIN para cada dimensão (benefícios, resultados e dificuldades) são apresentados nas Tabelas 1 a 3, conjuntamente com os agrupamentos formados e os respectivos Índices H de cada item. Também se incluem o número de respondentes e o percentual relativo das categorias "moderadamente importante" e "muito impor-

7 Para a análise da TRIN utilizou-se o pacote "mokken" do software estatístico R. Outras técnicas, como a Modelagem de Equaçôes Estruturais (SEM - Structural Equation Modeling), poderiam ser utilizadas, porém essas técnicas apresentam problemas, por exemplo, quando a relação entre os itens é não linear. A não linearidade dos dados, independentemente do uso de medidas de correlaçôes adequadas, pode resultar em uma incompatibilidade entre o modelo e os dados, como apontaram Ackerman et al. (2003). Outra abordagem possível seria o uso de técnicas de Análise de Cluster (Agrupamentos) aplicada às variáveis do questionário e não às unidades de observação. Contudo, a opção pela técnica de TRIN é bastante apropriada, visto que essa técnica foi criada especificamente para gerar escalas a partir de informaçōes coletadas por questionários e formada por vezes por diversas dimensōes, como no questionário do survey em estudo.

8 O resultado do Índice de Escalonamento encontra-se nas Tabelas 1 a 3. O Índice H é considerado forte se for $\geq 0,5$ e o limite inferior é de 0,3, como sugerido por Mokken (1971). Todos os índices foram superiores a esse limite inferior e a maioria dos itens apresentou $\mathrm{H}$ superior a 0,5 , portanto, nenhum item foi descartado. 
tante" para cada item. Os resultados geraram dois agrupamentos para os benefícios e três para as dimensões resultados e dificuldades.

TABELA 1

Agrupamentos para a dimensão "benefícios da interação"

\begin{tabular}{|c|c|c|c|c|}
\hline Agrupamentos & Itens & $\begin{array}{l}\text { Moderadamente } \\
\text { importante ou muito } \\
\text { importante (1) }\end{array}$ & $\%(2)$ & $\begin{array}{c}\text { Índice } \mathbf{H} \\
\text { (3) }\end{array}$ \\
\hline \multirow{5}{*}{$\begin{array}{l}\text { Benefícios } \\
\text { intangíveis }\end{array}$} & $\begin{array}{l}\text { Ideias para novos projetos de } \\
\text { cooperação }\end{array}$ & 502 & 82,0 & 0,84 \\
\hline & $\begin{array}{l}\text { Novas ideias de projetos de } \\
\text { pesquisa }\end{array}$ & 536 & 87,6 & 0,84 \\
\hline & $\begin{array}{l}\text { Intercâmbio de conhecimentos } \\
\text { ou informaçôes }\end{array}$ & 504 & 82,4 & 0,73 \\
\hline & Novas redes de relacionamento & 440 & 71,9 & 0,68 \\
\hline & Reputação & 430 & 70,3 & 0,65 \\
\hline \multirow{3}{*}{$\begin{array}{l}\text { Benefícios } \\
\text { tangíveis }\end{array}$} & $\begin{array}{l}\text { Equipamentos/ instrumentos } \\
\text { de uso compartilhado }\end{array}$ & 332 & 54,2 & 0,65 \\
\hline & $\begin{array}{l}\text { Recebimento de insumos para } \\
\text { as pesquisas }\end{array}$ & 429 & 70,1 & 0,65 \\
\hline & Recursos financeiros & 430 & 70,3 & 0,57 \\
\hline
\end{tabular}

Fonte: Survey da Pesquisa sobre Interações UE. Elaboração dos autores.

(1) Número de respondentes que consideraram o item moderadamente importante ou muito importante.

(2) Porcentagem de respostas sobre o total de respondentes (612).

(3) Índice $\mathrm{H}$ - Índice de escalonamento.

Como é possível verificar na Tabela 1, a aplicação da TRIN para os itens da dimensão dos benefícios permitiu identificar duas escalas, aqui chamadas de agrupamentos. O primeiro agrupamento foi denominado beneficios intangiveis, por abranger benefícios de ordem intelectual ou elementos tácitos, como a reputação e relacionamentos. Já o segundo, chamado de beneficios tangiveis, é composto por itens ligados à obtenção de ganhos materiais ou financeiros da interação para a pesquisa (como recursos financeiros; recebimentos de insumos para pesquisa e equipamentos/ instrumentos de uso compartilhado).

Os percentuais de respostas "muito importante" ou "moderadamente importante" dos itens agrupados nos beneficios intangiveis são superiores aos dos beneficios tangiveis, o que aponta que os grupos de pesquisa tendem a perceber como relativamente mais importantes os beneficios intangiveis oriundos da interação.

No que se refere à percepção dos grupos sobre a dimensão dos resultados esperados da interação, foram identificados três agrupamentos, apresentados na Tabela 2. 
TABELA 2

Agrupamentos para a dimensão "resultados da interação"

\begin{tabular}{l|l|c|c|c}
\hline \multirow{2}{*}{ Agrupamentos } & \multicolumn{1}{c|}{ Itens } & $\begin{array}{c}\text { Moderadamente } \\
\text { importante ou muito } \\
\text { importante(1) }\end{array}$ & \% (2) & $\begin{array}{c}\text { Índice } \\
\text { H (3) }\end{array}$ \\
\hline $\begin{array}{l}\text { Resultados de } \\
\text { conhecimento }\end{array}$ & Novas descobertas científicas & 375 & 61,3 & 0,66 \\
\hline \multirow{3}{*}{$\begin{array}{l}\text { Resultados } \\
\text { acadêmicos }\end{array}$} & Novos projetos de pesquisa & 522 & 85,3 & 0,66 \\
\hline & Formação de RH e estudantes & 522 & 85,3 & 0,65 \\
& Teses e dissertaçōes & 525 & 85,8 & 0,75 \\
& Publicaçóes & 501 & 81,9 & 0,75 \\
& Software & 187 & 30,6 & 0,61 \\
\hline \multirow{3}{*}{$\begin{array}{l}\text { Resultados de } \\
\text { inovação }\end{array}$} & Novos produtos e artefatos & 357 & 58,3 & 0,65 \\
& Novos processos industriais & 275 & 44,9 & 0,74 \\
& Melhoria de produtos & & 47,9 & 0,74 \\
& industriais & 293 & & \\
& Melhoria de processos & & 48,7 & 0,77 \\
& industriais & 298 & 43,8 & 0,61 \\
& Patentes & 268 & 20,3 & 0,63 \\
& Design & 124 & 25,0 & 0,68 \\
\hline
\end{tabular}

Fonte: Survey da Pesquisa sobre Interações UE. Elaboração dos autores.

(1) Número de respondentes que consideraram o item moderadamente importante ou muito importante.

(2) Porcentagem de respostas sobre o total de respondentes (612).

(3) Índice $\mathrm{H}-$ Índice de escalonamento.

É possível notar que dois agrupamentos da dimensão dos resultados estão mais associados às finalidades acadêmicas e, por isso, foram rotulados de resultados de conhecimento, relacionados às novas descobertas e novos projetos de pesquisa, e resultados acadêmicos, associados à formação de recursos humanos, às teses e dissertações, às publicações e à produção de software. Por fim, tem-se o agrupamento dos resultados de inovação, mais associados aos fins industriais, abarcando atividades de desenvolvimento de produtos e processos, além de patentes, design e spin-offs.

Novamente, percebe-se que os dois primeiros agrupamentos tendem a ser mais bem avaliados pelos grupos de pesquisa, o que pode apontar uma maior inclinação da academia para resultados intelectuais da interação, condizente com o esperado para as respostas de profissionais do mundo acadêmico.

Por último, ao se avaliar a percepção do grupo a partir das dificuldades, foram identificados três agrupamentos dos itens, apresentados na Tabela 3. 
TABELA 3

Agrupamentos para a dimensão "dificuldades da interação"

\begin{tabular}{|c|c|c|c|c|}
\hline Agrupamentos & Itens & $\begin{array}{l}\text { Moderadamente } \\
\text { importante ou muito } \\
\text { importante (1) }\end{array}$ & $\%(2)$ & $\begin{array}{c}\text { Índice } \\
\text { H (3) }\end{array}$ \\
\hline \multirow{5}{*}{$\begin{array}{l}\text { Dificuldades de } \\
\text { capacitação }\end{array}$} & $\begin{array}{l}\text { Falta de conhecimento nas empresas } \\
\text { das atividades realizadas nas } \\
\text { universidades/institutos de pesquisa }\end{array}$ & 372 & 60,8 & 0,68 \\
\hline & $\begin{array}{l}\text { Falta de conhecimento das } \\
\text { necessidades das empresas por parte } \\
\text { das universidades/ institutos de } \\
\text { pesquisa }\end{array}$ & 364 & 59,5 & 0,68 \\
\hline & $\begin{array}{l}\text { Falta de pessoal qualificado } \\
\text { para estabelecer um diálogo nas } \\
\text { universidades/ institutos de pesquisa }\end{array}$ & 294 & 48,0 & 0,63 \\
\hline & $\begin{array}{l}\text { Falta de pessoal qualificado para } \\
\text { estabelecer um diálogo nas empresas }\end{array}$ & 321 & 52,5 & 0,62 \\
\hline & Problema de confiabilidade & 278 & 45,4 & 0,59 \\
\hline \multirow{2}{*}{$\begin{array}{l}\text { Dificuldades } \\
\text { de orientação }\end{array}$} & Diferença de prioridades & 348 & 56,9 & 0,51 \\
\hline & $\begin{array}{l}\text { Divergência quanto ao prazo da } \\
\text { pesquisa }\end{array}$ & 244 & 39,9 & 0,51 \\
\hline \multirow{5}{*}{$\begin{array}{l}\text { Dificuldades } \\
\text { transacionais }\end{array}$} & Burocracia por parte da empresa & 287 & 46,9 & 0,40 \\
\hline & $\begin{array}{l}\text { Burocracia por parte da universidade/ } \\
\text { institutos de pesquisa (limites } \\
\text { institucionais) }\end{array}$ & 475 & 77,6 & 0,43 \\
\hline & Custeio da pesquisa & 388 & 63,4 & 0,37 \\
\hline & Direitos de propriedade & 251 & 41,0 & 0,43 \\
\hline & Distância geográfica & 172 & 28,1 & 0,35 \\
\hline
\end{tabular}

Fonte: Survey da Pesquisa sobre Interações UE. Elaboração dos autores.

(1) Número de respondentes que consideraram o item moderadamente importante ou muito importante.

(2) Porcentagem de respostas sobre o total de respondentes (612).

(3) Índice $\mathrm{H}$ - Índice de escalonamento.

No que diz respeito às respostas sobre as dificuldades da interação, por meio da TRIN foi possível identificar um agrupamento denominado dificuldades de capacitação, envolvendo itens associados à ausência de pessoal ou conhecimento qualificado, tanto na empresa quanto no grupo. Um segundo agrupamento agrega as dificuldades de orientação, que incluem as diferenças de prioridades e prazos entre os grupos e as empresas. Esse agrupamento está bastante associado às diferenças de orientação do mundo acadêmico e empresarial, como apontado por Merton (1973). Por fim, foi gerado um agrupamento denominado dificuldades transacionais, englobando um 
conjunto de elementos nos quais predominam as dificuldades para formalizar e legitimar a ocorrência da interação, associados ao que Tartari et al. (2012) chamaram de barreiras de Williamson.

Identificados e validados os agrupamentos para cada dimensão percebida, foi possível avaliar o impacto delas no número de interações dos grupos com as empresas, por meio da estimação de um modelo econométrico, que relaciona os agrupamentos criados e outras variáveis com a quantidade de interaçóes.

\section{Modelo estimado e resultados obtidos}

Após análise dos agrupamentos de itens, foi definido o modelo para analisar como as percepções dos grupos de pesquisa acadêmica sobre resultados, benefícios e dificuldades esperados das interações influenciam o número de interações que eles realizam com empresas.

Para tanto, a amostra utilizada é composta por 612 grupos de pesquisa que assinalaram o número de interações com empresas e suas percepções gerais sobre essas interaçōes em um survey. Todos os grupos estavam vinculados a programas de pós-gradação de diversas áreas do conhecimento e de todas as regiões do Brasil.

Assim, o modelo proposto permite relacionar a quantidade de interaçôes realizadas pelos grupos com os agrupamentos gerados (identificados pela TRIN), bem como relacionar a qualidade da pesquisa acadêmica, o tamanho do grupo de pesquisa e o tamanho departamento ao qual o grupo pertence com o número de interações realizadas.

Com o intuito de controlar o nível de qualificação do entorno do grupo de pesquisa e o grau de adensamento das regiōes, foram adicionadas ao modelo duas variáveis: a qualificação da mão de obra e a densidade urbana da microrregião na qual o grupo está inserido. A inclusão dessas variáveis controla os efeitos das condições externas ao grupo que podem influenciar os resultados do modelo.

Por fim, também foram consideradas dummies para representar as diferentes áreas de conhecimento. Espera-se que o número de interações dos grupos de pesquisa tenha comportamento distinto de acordo com as áreas de conhecimento, tema já apresentado em trabalhos como Bekker e Freitas (2008) e Meyer-Krahmer e Schmoch (1998). Tal suposição é baseada na ideia de que existem diferenças verificadas no papel das disciplinas acadêmicas no suporte às atividades inovativas das empresas. Segundo Metcalfe (2003), por exemplo, algumas áreas, como as Engenharias, tendem a gerar conhecimentos mais aplicados do que as Ciências Naturais. Schartinger et 
al. (2001) e D’Este e Iammarino (2010) também apontaram a existência de padrões distintos entre as áreas de conhecimento.

O modelo estimado é representado pela seguinte forma funcional:

$$
\begin{gathered}
N^{5} I n t=\text { Beneficios }_{+} \text {Resultados } \text { Dificuldade }+ \text { Qualidade }_{\text {depto }}+\text { Tamanho }_{\text {depto }}+ \\
\text { Tamanho } \\
\text { grupo }+ \text { Controles }
\end{gathered}
$$

O Quadro 1 apresenta o resumo de cada variável com sua respectiva proxy e fonte. Os resultados do modelo proposto encontram-se na Tabela 4.

QUADRO 1

\begin{tabular}{|c|c|c|}
\hline Variáveis & Descrição & Fonte \\
\hline $\mathrm{N}^{\circ}$ Int & $\begin{array}{l}\text { Número de interaçôes com empresas relatadas pelo } \\
\text { grupo }\end{array}$ & Survey \\
\hline Benefícios & $\begin{array}{l}\text { Dummies para cada agrupamento igual a } 1 \text { se } \\
\text { mediana das respostas dos itens for maior ou igual } \\
\text { a } 3 \text { (1) }\end{array}$ & \multirow{3}{*}{$\begin{array}{l}\text { Elaboração própria a } \\
\text { partir de TRIN com } \\
\text { dados do survey }\end{array}$} \\
\hline Resultados & $\begin{array}{l}\text { Dummies para cada agrupamento igual a } 1 \text { se } \\
\text { mediana das respostas dos itens for maior ou igual } \\
\text { a } 3 \text { (1) }\end{array}$ & \\
\hline Dificuldades & $\begin{array}{l}\text { Dummies para cada agrupamento igual a } 1 \text { se } \\
\text { mediana das respostas dos itens for maior ou igual } \\
\text { a } 3 \text { (1) }\end{array}$ & \\
\hline $\begin{array}{l}\text { Qualidade do } \\
\text { departamento }\end{array}$ & $\begin{array}{l}\text { Média da nota atribuída aos programas de pós- } \\
\text { graduação da área de avaliação em que o grupo de } \\
\text { pesquisa está inserido }\end{array}$ & Capes, 2007 \\
\hline $\begin{array}{l}\text { Tamanho do } \\
\text { departamento }\end{array}$ & $\begin{array}{l}\text { Número de docentes na IES do grupo de pesquisa } \\
\text { no ano de } 2006\end{array}$ & Capes, 2006 \\
\hline $\begin{array}{l}\text { Tamanho do } \\
\text { grupo }\end{array}$ & Total de integrantes do grupo de pesquisa & Survey \\
\hline Capital humano & $\begin{array}{l}\text { Pessoas empregadas na indústria de transformação } \\
\text { com nível superior na microrregião em que o grupo } \\
\text { está localizado }\end{array}$ & RAIS, 2006. \\
\hline Densidade urbana & $\begin{array}{l}\text { Densidade populacional urbana da microrregião } \\
\text { (pop. urbana por área urbanizada) em que o grupo } \\
\text { está localizado }\end{array}$ & $\begin{array}{l}\text { IBGE, 2007; Embrapa, } \\
2000\end{array}$ \\
\hline Área & Dummies para áreas do conhecimento & Survey \\
\hline
\end{tabular}

Descrição das variáveis utilizadas no estudo

(1) As respostas "sem importância", "pouco importante", "moderadamente importante" e "muito importante" foram categorizadas na escala de 1 a 4 , respectivamente. 
TABELA 4

Estimativas dos coeficientes - Regressão Binomial Negativa

\begin{tabular}{cll}
\hline \multirow{2}{*}{ Benefícios } & Intangíveis & $0,3545(0,1395)^{*}$ \\
& Tangíveis & $0,0376(0,1039)$ \\
\hline \multirow{2}{*}{ Resultados } & Conhecimento & Acadêmicos \\
& Inovação & $0,4850(0,1013)^{* * *}$ \\
& Capacitação & $-0,0159(0,1086)$ \\
Dificuldades $\quad$ Orientação & $-0,0358(0,1011)$ \\
\hline Tamanho do departamento & $-0,1198(0,0910)$ \\
Qualidade do departamento & $0,0373(0,0987)$ \\
Tamanho da equipe de pesquisa & $-0,4013(0,0953)^{* * *}$ \\
Capital humano & $0,0011(0,0004)^{*}$ \\
Densidade pop. urbana & $0,0999(0,0421)^{*}$ \\
Dummies para áreas de conhecimento & $0,0079(0,0015)^{* * *}$ \\
Constante & $1,46 \mathrm{e}-08(1,65 \mathrm{e}-07)$ \\
\hline
\end{tabular}

Número de Observações: 612

$\begin{array}{lll}\mathrm{LR} \operatorname{chi} 2(17)=326,23 & \text { Prob }>\text { chi } 2=0,0000\end{array}$

Log likelihood $=-1426,3576 \quad D^{2} R^{2}=0,8195^{\#}$

Fonte: Survey da Pesquisa sobre Interações UE; Capes; RAIS, IBGE; Embrapa. Elaboração dos autores.

${ }^{* * *} \mathrm{p}<0.1 \%$; ${ }^{* *} \mathrm{p}<1 \%$; ${ }^{*} \mathrm{p}<5 \%$; erro-padrão em parênteses.

\# - O DP R ${ }^{2}$ é uma medida da qualidade do ajuste mais apropriada a modelo binomial negativo, como indicado por Cameron

e Windmeijer (1996).

Entre os resultados encontrados no modelo, destacam-se os coeficientes positivos e significantes para as variáveis dos agrupamentos de benefícios intangiveis e resultados de conhecimento, e negativo e significante para as dificuldades transacionais.

É possível apontar que os beneficios intangiveis influenciaram positivamente o número de interaçôes que os grupos de pesquisa realizaram com empresas, resultado alinhado com o encontrado por Garret-Jones et al. (2010). Este agrupamento inclui uma série de ganhos associados à própria natureza da atividade acadêmica, como a difusão e produção de conhecimento (ideias para novos projetos e redes de relacionamento) e outros comportamentos intrínsecos ao seu funcionamento, como a busca de reputação acadêmica. Ou seja, a partir desses resultados, é possível dizer que as expectativas dos grupos de pesquisa relacionadas ao aumento dos fluxos e 
da produção de conhecimento e, também, da melhoria da reputação fomentam suas interaçôes.

Apesar de outros trabalhos evidenciarem a importância dos beneficios tangiveis no maior número de interações, principalmente como meio de obtenção de recursos (TARTARI; BRESCHI, 2012), o resultado do modelo não permite inferir uma relação entre beneficios tangiveis e a quantidade de interaçôes. Com vista ao argumento de Tartari e Breschi (2012), é possível que esse resultado esteja associado ao fato de que alguns pesquisadores acadêmicos tendem a reportar como menos relevantes os benefícios tangíveis, grande parte por receio de juízo negativo de alguns pares do meio acadêmico.

No que se refere aos agrupamentos relacionados à percepção dos resultados da interação U-E, pode-se verificar que os resultados de conhecimento apresentam efeito positivo e significante no número de interações dos grupos. Ou seja, a percepção por parte dos grupos de que a interação possa gerar novas descobertas científicas e novos projetos de pesquisa é um fator que promove as interações. Já os resultados acadêmicos e resultados de inovação apresentam efeitos não significantes no modelo. Dessa maneira, pode-se supor que os pesquisadores interagem mais com empresas em busca não de publicações imediatas ou outros resultados acadêmicos, mas sim de abertura de outras oportunidades de pesquisa. ${ }^{?}$

Esse resultado contrasta com a visão de que a interação da universidade com a empresa pode desviar a essência da missão acadêmica. $\mathrm{O}$ resultado aqui apontado mostra que as expectativas de novas descobertas científicas ou projetos de pesquisa impulsionam a interação com empresas. Nesse sentido, ainda que a interação envolva segredos e atrasos da publicação atrelados aos resultados da interação, a perspectiva de que novas ideias surjam da interação é fator preponderante para os grupos interagirem mais.

Os coeficientes de resultados de conhecimento e beneficios intangiveis possuem uma expressiva semelhança com o achado por D’Este e Perkmann (2011), que verificaram um efeito positivo das motivaçôes de aprendizado (learning) em interaçōes dos grupos acadêmicos, do tipo pesquisa conjunta, pesquisa contratada e consultoria. Ou seja, destaca-se que a busca de benefícios e resultados relativos a

9 Ainda que o questionário aplicado aos grupos avalie as percepçóes como um todo, pode-se argumentar que os respondentes seriam influenciados por suas experiências recentes de interação. Esse fato poderia gerar um potencial problema de endogeneidade na dimensão resultados por estar mais associada ao produto final das interações. Porém, mesmo que não seja possível com exatidão mostrar a inexistência de endogeneidade, deve-se apontar que as variáveis das percepçōes foram criadas de modo binário (dummies para as categorias "muito importante" e "importante") e não em valor absoluto, o que reduz esse problema potencial. 
ideias e projetos de pesquisa é importante motivador para que os grupos realizem mais interações com empresas.

Para os agrupamentos ligados às dificuldades percebidas, verificou-se que as dificuldades de capacitação e dificuldades de orientação não apresentam significância. Portanto, não se encontrou um resultado similar ao obtido por Tartari et al. (2012), que apontaram que, na Itália, dificuldades de orientação (obstáculos de orientação para os autores) seriam as principais barreiras para a ocorrência de interaçōes entre universidade e empresas.

A variável que representa as dificuldades transacionais, relacionadas aos processos para formalizar e legitimar a ocorrência da interação, possui coeficiente negativo e significante. Isso indica que os grupos que percebem maiores dificuldades envolvendo a burocracia, o custeio da pesquisa, os diretos de propriedade ou a distância geográfica das partes envolvidas tendem a interagir menos. Nesse caso, é interessante ter em conta que a estrutura institucional acadêmica brasileira possui algumas singularidades diante de outros países analisados pela literatura. No Brasil, a pesquisa universitária está fortemente concentrada em universidades e institutos de pesquisa públicos (GREGOLIN et al., 2005), o que pode sugerir um indicativo do reforço da percepção de barreiras de ordem institucional e burocrática. Esse resultado pode indicar a necessidade de orientar as políticas públicas que buscam fomentar as interações no sentido de reduzir esses entraves percebidos na formalização das interações entre grupos de pesquisa e empresas.

A relevância das dificuldades transacionais pode estar associada à presença do item burocracia da universidade nesse agrupamento, uma vez que foi o item apontado pelo maior número de respondentes como muito ou moderadamente importante. Para verificar se essa relação permanece sem esse elemento, realizou-se um teste adicional, com a exclusão desse item no agrupamento. $\mathrm{O}$ modelo adicional estimado aponta novamente que as dificuldades transacionais, representadas apenas pelos demais itens (custeio da pesquisa, direitos de propriedade e distância geográfica), também apresentam efeitos negativos no número de interações.

No que diz respeito às características dos grupos, as três variáveis analisadas mostraram-se significantes. Como esperado, os resultados do modelo empírico expressam uma relação positiva entre o tamanho do grupo, o tamanho do departamento e a qualidade da pesquisa acadêmica com a quantidade de interações do grupo.

Assim, em relação à qualidade da pesquisa, nota-se que grupos que possuem maior qualificação acadêmica tendem a interagir com mais empresas, comparativamente aos seus pares com desempenho inferior, o que corrobora com o apresentado 
em outros trabalhos (BISHOP et al., 2011; D’ESTE; IAMMARINO, 2010). Grupos situados em departamentos maiores tendem a ser mais interativos. Isto pode evidenciar, como sugerido por Schartinger et al. (2001), que grupos alocados em departamento maiores sabem tirar proveito da sua melhor estrutura e da disponibilidade de recursos humanos. Observa-se também que grupos maiores estão associados a um maior número de interações. Por fim, não foi possível tirar conclusões sobre as diferentes áreas de conhecimento e as características do entorno onde o grupo está localizado (capital humano e densidade urbana), pois não foram significativos para explicar o número de interaçôes.

\section{Conclusões e implicações}

Este trabalho busca dar novos subsídios para a discussão sobre interações entre universidades e empresas no Brasil, analisando como as percepções dos grupos de pesquisa acadêmica sobre resultados, benefícios e dificuldades esperados das interações influenciam o número de interaçôes que eles realizam com empresas. Essa discussão também está presente em trabalhos como Tartari e Breschi (2012), Tartari et al. (2012) e Arza e Vazquez (2010).

Dessa forma, o artigo procura contribuir com dois pontos: primeiro, por meio da realização de uma análise não ad hoc das distintas percepções dos grupos de pesquisa sobre os benefícios, resultados e dificuldades relacionados às interaçôes; e, segundo, com a estimação de um modelo que buscou avaliar o quanto essas dimensões afetam o número total de interações dos grupos de pesquisa, controlados diversos fatores, como a qualidade da pesquisa acadêmica.

Da primeira análise foi possível validar e explorar os padrões de respostas dos itens do questionário referentes às dimensões de benefícios, resultados e dificuldades percebidos pelos grupos de pesquisa, criando assim agrupamentos: dois para a dimensão benefícios (tangíveis e intangíveis); três para a dimensão resultados (de conhecimento; acadêmico e de inovação); e outros três para as dificuldades (de capacitação; de orientação e transacionais). Esse passo é importante por se tratar de agrupamentos não arbitrários que tornam possíveis a aplicação do modelo de forma adequada, com estimativas cujos parâmetros reflitam de maneira apropriada o objetivo deste trabalho.

A estimação do modelo indica alguns resultados relevantes. Primeiramente, observa-se que os grupos acadêmicos que percebem benefícios intangíveis e resultados de conhecimento como mais importantes tendem a ser mais interativos. Desses dois 
achados, pode-se indicar que os ganhos associados à natureza da atividade acadêmica, como difusão e produção de conhecimento e outras realidades intrínsecas ao seu funcionamento, favorecem as interações por parte dos grupos. Achado, por sinal, em linha com o encontrado por D’Este e Perkmann (2011) e Garret-Jones et al. (2010).

Do ponto de vista das dificuldades para as interações, nota-se que as dificuldades transacionais influenciam negativamente as interaçóes. Como essas dificuldades estão relacionadas aos processos para formalizar e legitimar a ocorrência da interação, conclui-se que os grupos que percebem maiores dificuldades envolvendo a burocracia, o custeio da pesquisa, os direitos de propriedade ou a distância das partes envolvidas tendem a interagir menos.

Esses resultados apresentam interessantes implicações para o campo de políticas públicas. $\mathrm{O}$ fato de que os resultados de conhecimento e os beneficios intangiveis levam a um maior número de interações pode ser um indicativo de que as interações conduzem a novas pesquisas acadêmicas, novas ideias e maior intercâmbio de conhecimento, o que sugere um impacto positivo e expressivo na interação com empresas na pesquisa universitária. Ainda que a interação possa envolver divergências entre a natureza das atividades desenvolvidas entre universidades e empresas, as perspectivas desses benefícios e resultados para os pesquisadores respondentes são estimuladoras para tais relacionamentos. Do ponto de vista dos formuladores de políticas, esse achado pode apontar que a promoção dessas relações seria uma maneira a mais de impulsionar as finalidades de geração de conhecimento na academia.

Por outro lado, o resultado de que as dificuldades transacionais impactam negativamente no número de interaçôes dos grupos pode indicar a necessidade de simplificar procedimentos para formalizar e legitimar as ocorrências de interaçóes. Porém, para obterem sucesso, essas medidas têm que levar em conta que, no Brasil, a maioria das interações ocorre com grupos de pesquisa de universidades públicas.

Para desenvolvimentos futuros, parece oportuno o aprofundamento nos efeitos dessas dimensões (benefícios, resultados e dificuldades) a partir de um conjunto maior de dados, que inclua também características da empresa que interage (setor, tamanho, intensidade de P\&D) e da interação.

\section{Referências bibliográficas}

ABRAMOVSKY, L.; HARRISON, R.; SIMPSON, H. University research and the location of business R\&D. The Economic Journal, n. 117, p. 114-141, 2007. 
ACKERMAN, T. A.; GIERL, M. J.; WALKER, C. M. Using multidimensional item response theory to evaluate educational and psychological tests. Educational Measurement: Issues and Practice, v. 22, n. 3, p. 37-51, 2003.

ARZA, V. Channels, benefits and risks of public-private interactions for knowledge transfer: conceptual framework inspired by Latin America. Science and Public Policy, v. 37,n. 7, p. 473-484, 2010.

ARZA, V.; VAZQUEZ, C. Interactions between public research organizations and industry in Argentina. Science and Public Policy, v. 37, n. 7, p. 499-511, 2010.

AUDRETSCH, D. B.; BOZEMAN, B.; COMBS, K. L.; FELDMAN, M.; LINK, A. N.; SIEGEL, D. S. et al. The economics of science and technology. The Journal of Technology Transfer, v. 27, n. 2, p. 155-203, 2002.

BEKKERS, R.; FREITAS, I. M. B. Analysing knowledge transfer channels between universities and industry: To what degree do sectors also matter? Research Policy, n. 37, p. 1837-1853, 2008.

BISHOP, K.; D’ESTE, P.; NEELY, A. Gaining from interactions with universities: Multiple methods for nurturing absorptive capacity. Research Policy, n. 40, p. 30-40, 2011.

CAMERON, A. C.; WINDMEIJER, F. A. G. R-squared measures for count data regression models with applications to health-care utilization.Journal of Business \& Economic Statistics, v. 14, n. 2, p. 209-220, 1996.

CHAVES, C. V.; CARVALHO, S. S. M.; SILVA, L. A.; TEIXEIRA, T. C.; BERNARDES, P. The point of view of firms in Minas Gerais about the contribution of universities and research institutes to R\&D activities. Research Policy, n. 41, p. 1683-1695, 2012.

COHEN, W.; NELSON, R.; WALSH, J. Links and impacts: the influence of public R\&D on industrial research. Management Science, n. 48, p. 01-23, 2002.

DASGUPTA, P.; DAVID, P. A. Toward a new economics of science. Research Policy, n. 23, p. 487-521, 1994.

D'ESTE, P.; IAMMARINO, S. The spatial profile of university-business research partnerships. Papers in Regional Science, v. 89, n. 2, p. 335-351, 2010.

D'ESTE, P.; PERKMANN, M. Why do academics engage with industry? The entrepreneurial university and individual motivations. Journal of Technological Transfer, n. 36, p. 316-339, 2011.

DE FUENTES, C.; DUTRÉNIT, G. Best channels of academia-industry interaction for long-term benefit Research Policy, v. 41, n. 9, p. 1666-1682, 2012. 
DURANTON, G.; PUGA, D. Nursery cities: urban diversity, process innovation, and the life cycle of products. The American Economic Review, v. 91, n. 5, p. 1454-1477, 2001.

FERNANDES, A. C.; CAMPELLO DE SOUZA, B.; STAMFORD SILVA, A.; SUZIGAN, W.; CHAVES, C.; ALBUQUERQUE, E. Academy-industry links in Brazil: evidence about channels and benefits for firms and researchers. Science and Public Policy, v. 37, n. 7, p. 485-498, 2010.

FREEMAN, C. The "National System of Innovation" in historical perspective. Cambridge Journal of Economics, v. 19, n. 1, p. 5-24, 1995.

GARRETT-JONES, S.; TURPIN, T.; DIMENT, K. Managing competition between individual and organizational goals in cross-sector research and development centres. The Journal of Technology Transfer, v. 35, n. 5, p. 527-546, 2010.

GREGOLIN, J.; HOFFMANN, W.; FARIA, L.; QUONIAM, L.; QUEYRAS, J. Capítulo 5 - Análise da produção científica a partir de indicadores bibliométricos. In: LANDI, F. R. (Org.). Indicadores de ciência, tecnologia e inovação em São Paulo 2004. São Paulo: Fapesp, 2005.

KLEVORICK, A.; LEVIN, R.; NELSON, R.; WINTER, S. On the sources and significance of inter-industry differences in technological opportunities. Research Policy, n. 24, p. 185-205, 1995.

LAURSEN, K.; REICHSTEIN, T.; SALTERS, A. Exploring the effect of geographical proximity and university quality on university-industry collaboration in the United Kingdom. Regional Studies, v. 45, n. 4, p. 507-523, 2011.

MANSFIELD, E.; LEE, J. The modern university: contributor to industrial innovation and receipt of industrial R\&D support. Research Policy, n. 25, p. 1047-1058, 1996.

MERTON, R. K. The sociology of science. Theoretical and empirical investigations. Chicago: University of Chicago Press, 1973.

METCALFE, S. Equilibrium and evolutionary foundations of competition and technology policy: new perspectives on the division of labour and the innovation process. Revista Brasileira de Inovação,v. 2, n. 1, p. 111-146, 2003.

MEYER-KRAHMER, F.; SCHMOCH, U. Science-based technologies: university-industry interactions in four fields. Research Policy, v. 27, n. 8, p. 835-851, 1998.

MOKKEN, R. J. A theory and procedure of scale analysis: With applications in political research. New York: Walter de Gruyter, 1971.

MOWERY, D. C.; NELSON, R. R.; SAMPAT, B. N.; ZIEDONIS, A. A. Ivory tower and industrial innovation - University-industry technology transfer before and after the BayhDole Act. Stanford University Press: Palo Alto, CA, 2004. 
NELSON, R. Sources of economic growth. Cambridge: Harvard University, 1996.

PERKMANN, M.; KING, Z.; PAVELIN, S. Engaging excellence? Effects of faculty quality on university engagement with industry. Research Policy, n. 40, p. 539-552, 2011.

PERKMANN, M.; WALSH, K. The two faces of collaboration: impacts of university-industry relations on public research. Industrial and Corporate Change, v. 18, n. 6, p. 1033-1065, 2009.

RAPINI, M. S.; ALBUQUERQUE, E. M.; CHAVES, C. V.; SILVA, L. A.; SOUZA, S. G. A.; RIGHI, H. M.; CRUZ, W. M. S. University-industry interactions in an immature system of innovation: evidence from Minas Gerais, Brazil. Science and Public Policy, n. 36, p. 373-386, 2009.

ROSENBERG, N. Why do firms do basic research (with their own money)? Research Policy, v. 19,1990

ROSENBERG, N.; NELSON, R. American universities and technical advance in industry. Research Policy, v. 23, n. 3, p. 323-348, 1994.

SCHARTINGER, D.; SCHIBANY, A.; GASSLER, H. Interactive relations between universities and firms: empirical evidence for Austria. Journal of Technology Transfer, n. 26, p. 255-268, 2001.

STORPER, M.; VENABLES, A. J. Buzz: face-to-face contact and the urban economy. Journal of Economy Geography, v. 4, n. 4, p. 351-370, 2004.

SUZIGAN, W.; ALBUQUERQUE, E.; GARCIA, R.; RAPINI, M. University and industry linkages in Brazil: some preliminary and descriptive results. Seoul Journal of Economics, v. 22, n. 4, p. 591-611, 2009.

SUZIGAN, W.; ALBUQUERQUE, E. M. The underestimated role of universities for the Brazilian system of innovation. Brazilian Journal of Political Economy, v. 31, n. 1, p. 3-30, 2011.

TARTARI, V.; BRESCHI, S. Set them free: scientists' evaluations of the benefits and costs of university-industry research collaboration. Industrial and Corporate Change, v. 21, n. 5, p. 1117-1147, 2012.

TARTARI, V.; SALTER, A.; D'ESTE, P. Crossing the rubicon: exploring the factors that shape academics' perceptions of the barriers to working with industry. Cambridge Journal of Economics, v. 36, n. 3, p. 655-677, 2012.

TORNQUIST, K. M.; KALLSEN, L. A. Out of the ivory tower: characteristics of institutions meeting the research needs of industry. Journal of Higher Education, v. 65, n. 5, p. 523-539, 1994.

WILLIAMSON, O. E. The economic institutions of capitalism. New York: Free Press, 1987. 
Influência das percepções dos grupos de pesquisa nas interações com empresas

\section{ANEXO}

Estatística descritiva das variáveis contínuas utilizadas

\begin{tabular}{lrrrr}
\hline \multicolumn{1}{c}{ Variáveis } & Média & $\begin{array}{c}\text { Desvio- } \\
\text { padrão }\end{array}$ & Mínimo & Máximo \\
\hline Número de interações & 4,5 & 19,9 & 0,0 & 400,0 \\
Tamanho do departamento & 71,1 & 106,9 & 8,0 & 759,0 \\
Qualidade do departamento & 4,5 & 1,0 & 2,5 & 7,0 \\
Tamanho da equipe de pesquisa & 23,6 & 28,4 & 2,0 & 600,0 \\
Capital humano - Sup. & 237624,4 & 308079,2 & 3454,0 & 988203,0 \\
Densidade demográfica & 2243,1 & 2549,9 & 224,2 & 13838,2 \\
\hline
\end{tabular}

Fonte: Survey da Pesquisa sobre Interações UE; Capes; RAIS, IBGE; Embrapa. Elaboração dos autores. 
